\title{
A Review of Recognition Techniques in ALPR Systems
}

\author{
Sahil Khokhar \\ Department of Electronics and Communication \\ DCRUST, Murthal, Sonepat, India
}

\author{
Pawan Kumar Dahiya \\ Department of Electronics and Communication \\ DCRUST, Murthal, Sonepat, India
}

\begin{abstract}
'Automatic License Plate Recognition (ALPR)' is the process of locating \& extracting plate information from images or videos. The extracted information is essential for several everyday applications, ranging from automated payment services (parking, toll roads payment collection, etc.) to more critical applications, like border security and traffic surveillance. The format of license plate varies from region to region, so the ALPR systems also tend to be region-specific. The paper presents a state-of-the-art review of earlier work done in this field of technology, especially, the recognition technique.
\end{abstract}

\section{Keywords}

Automatic License Plate Recognition (ALPR), Support Vector Machine (SVM), Artificial Neural Network (ANN).

\section{INTRODUCTION}

Automatic License Plate Recognition (ALPR) system is the technique used to extract the License Plate of a vehicle from an image. The main applications of the technologies include its use at Toll Booths, Car parkings etc. for faster service, at Traffic Signals to automatically detect vehicles jumping the red light and used for Traffic Control to help monitor the movement and flow of vehicles around the road network [13].

The ALPR system can be composed of five stages [1]. The first is to acquire the image of the vehicle. This can be done either with a live feed camera or from a database storage. The second step is to enhance the image for the extraction of the license plate. The third step is to locate the license plate in the image. The forth step is to segment each individual character present in the plate. The final step is to recognize each segmented character.

The purpose of this paper is to provide the survey of basic ALPR process with main focus on the recognition techniques used and the problems encountered by these techniques.

The remainder of the paper is organized as follows. In Section 2, the steps involved in ALPR are explained. In Section 3, the recognition techniques used are discussed. Section 4 discusses the problems associated with the existing technologies. In Section 5 and 6 , the future scope of work and the conclusion is discussed respectively.

\section{AUTOMATIC LICENSE PLATE RECOGNITION}

In this section, the steps involved in the ALPR process are briefly discussed. The ALPR process consists of 5 steps as mentioned in [1].

First the image with the license plate is acquired and then enhance it to facilitate the extraction of the license plate. The enhancement usually involves RGB to gray scale conversion, noise removal and contrast enhancement [1], [2].

Then the region of interest, i.e. the license plate, is localized. In [7], after the image enhancement, the license plate localization has four more steps namely, Edge detection, Separating objects from background, Finding connected component and Candidate selection. For edge detection, any edge detecting method can be used like Canny's method, Sobel or Prewitt detector. Then the 8-connectivity pixel are converted into 4-connectivity pixels using diagonal fill. Then the connected objects are investigated using 4 or 8 -ary connectivity. At last, the likely candidates are selected based on their properties like area, Range of length, aspect ratio and width and region intensity.

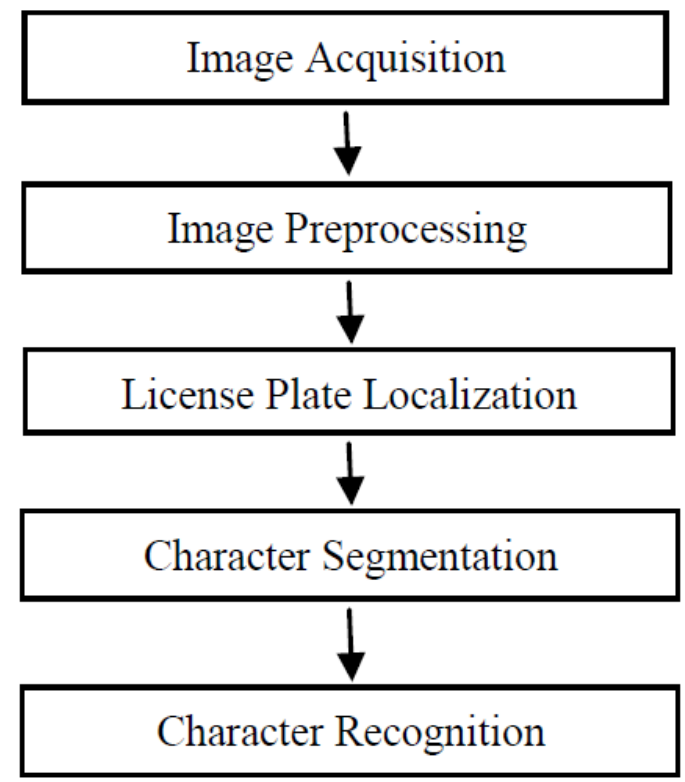

Figure 1: Steps involved in ALPR [6].

Each character is then segmented from the extracted license plate. In [8] and [9], it is done by first converting the license plate into a binary image. Then an 8-connected component analysis is used and noise is also removed. At last, the characters are segmented based on the valleys present in the histogram along a horizontal line through the characters.

The final step of the ALPR is the character segmentation. As mention in [10], there are two approaches that can be used for this purpose. The first is Template matching, in which a cross correlation between the segmented character and its stored template is performed. The second approach is feature matching, in which a comparison between the features extracted from the segmented image and pre-stored features of a character is performed.

\section{RECOGNITION TECHNIQUES}

The recognition techniques can be broadly divided into two type, i.e. template matching and feature matching. In template matching, the similarities between an extracted character and each of the templates are measured and the template whose correlation coefficient with the extracted character is the highest is considered as target [15]. Whereas in feature matching, first the features of the extracted character are extracted and then any classifier, such as Support Vector 
Machine (SVM), Artificial Neural Network (ANN) etc., can be used to recognize the characters [5].

In [3], SVM is described as a pattern recognizer that can classify the data provided, without taking any assumptions about the underlying process that was used to obtain the observations. SVM separates different classes by using hyperplanes. Out of many hyperplanes, the optimal one generalizes well in comparison to others.

In [4], two approaches are described to apply SVM to multiclass problems. The first one is called 'One Vs. All' (OVA), it uses a set of binary classifiers, each of which is trained to separate one class from the rest. The second one is called 'One Vs. One' (OVO), in which a series of classifiers are applied to each pair of classes, and only the most commonly computed class' label is kept for each case.

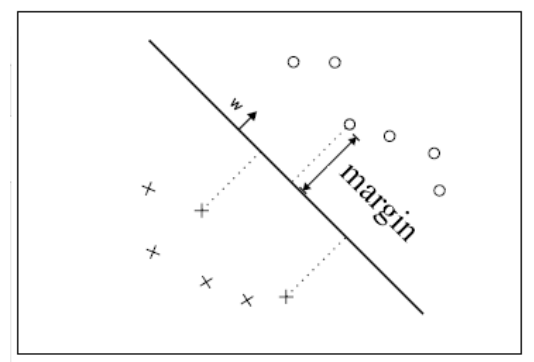

(a)

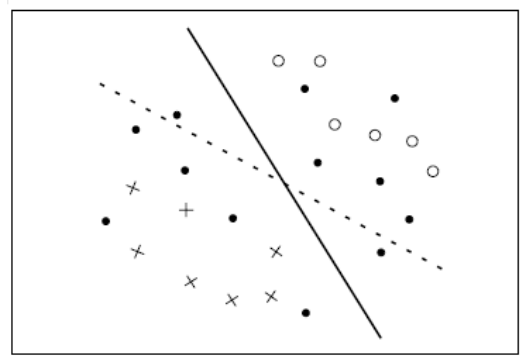

(b)

Figure 2: Optimal Hyperplane vs. Other Hyperplane [12].

In [3] and [4], SVM is used for the character recognition. In [4], it was observed that 'one against all' (OVA) obtained better accuracy as compared with the 'one against one' (OVO) method. The SVM technique works well in dark and poor weather conditions.

In [6], the working of ANN is described to be based on how human brain functions. In a neural network, some neurons interfaces to the real world to receive its input, whereas some neurons provide the world with the networks output. All the other neurons are hidden from the view. But a successful neural network is not just a random connection of neurons. One of the easiest ways to design a structure is to group these neurons into layers.

In [6], two methods of training an ANN are mentioned. The first is the supervised method, in which both the inputs and outputs are provided after that the inputs are processed and compared against the desired output. In unsupervised method, only the inputs are provided to the network, the system has to decide for itself, what features will be used to group the input data. But, at the present time, unsupervised learning is not well understood.

In [5], ANN is used and the accuracy of neural network is compared with the accuracy of KMEANS technique, in which the neural network clearly came out on top with $95.6 \%$ accuracy.
In [14], the efficiency of character recognition through template matching and Probability Neural Network (PNN) is tested and the results of two techniques are compared. Comparing the two techniques, it can be noted that Template Matching algorithm produced slightly better results.

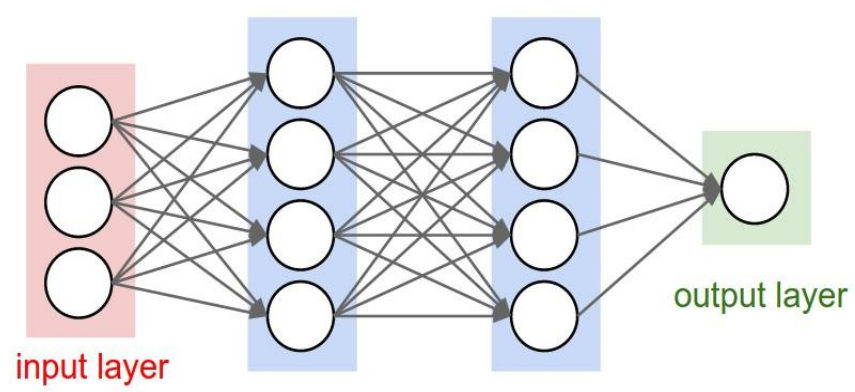

hidden layer 1 hidden layer 2

Figure 3: Simple Neural Network Diagram [17].

Table 1: Comparison between Template Matching and PNN [14].

\begin{tabular}{|c|c|}
\hline Method & Success Rate \\
\hline Template Matching & $80.81 \%$ \\
\hline PNN & $80.06 \%$ \\
\hline
\end{tabular}

In [16], a hybrid of K-Nearest Neighbour and SVM is used for character recognition. First the character is recognized via $\mathrm{KNN}$ technique. Then if the recognized character is similar to any other character (such as K and X) then the SVM is used for the final verdict.

\section{PROBLEMS WITH EXISTING TECHNOLOGIES}

The problems with the existing technology starts with the step of the ALPR. As stated in [4] and [8], the image acquired must have high enough quality so that the characters on the license plate are clearly visible and the illumination conditions must also be right, the process will fail if the illumination is too low or too high. Also with real time applications, the problem of motion blur is also present.

In [11], it is mentioned that during the license plate localization phase, some false positives may occur if something in the image has somewhat similar features to the license plate. Also some false negatives may occur if the license plate is not been presented in an expected way in an image, for eg. the license plate is tilted more than expected.

In [10], it is pointed out that the recognition techniques may not function properly if they are not well trained with a large enough sample size. Also some of the similar looking characters are mistaken for each other, some of the pairs include $(0-\mathrm{O}),(8, \mathrm{~B}),(\mathrm{K}-\mathrm{X})$ etc. Out of these pairs the first two can be distinguished if there is a prior knowledge of position of alphabets and digits, but the confusion between two similar looking alphabets or two similar looking digits is more difficult to solve.

\section{SCOPE OF FUTURE WORK}

The Indian license plate is standardized, i.e. the first three characters are country code (IND), followed by two letter are state code, followed by the two digit district code, followed by one/two/three letter series code and at last the four digit registration number. This information can be used to 
minimize the errors between similar looking alphabets and digits like $\mathrm{B} \& 8$ and $\mathrm{O} \& 0$.

ALPR system should be able to process colours as much information can be gathered from that, like the colour of the license plate can used to identify the type of vehicle, i.e. private vehicles have white backgrounded license plate, commercial vehicles have yellow and diplomatic vehicles have red coloured license plate.

An effective ALPR system should have capability to recognise multi-style plates i.e. plates with different fonts and formats.

To reduce the effect of motion blur, high speed cameras can be used where vehicles are expected to have high speed like on the highways.

For video based ALPR, where the success rate is highly affected due to motion blur, the system should be able to recognise different parts of the plate from different frames and be able to patch it together to successfully recognise the license plate

\section{CONCLUSION}

A very high degree of accuracy is needed in the process of vehicle license plate recognition when working on a very busy road or parking which may not be possible to attain that level of accuracy manually as a human being gets fatigued due to monotonous nature of the job and cannot keep track of the vehicles when there is rush, without causing some sort of blockage. ALPR can significantly reduce the manpower needed in some areas like toll booths, traffic lights, etc. It can automate the process of recognizing vehicles.

The standardization of license plates has made the job of ALPR easier. The confusion between similar looking alphabets and digits can be avoided by this as the position of numbers and alphabets is known in advance.

It needs high resolution input with proper illumination condition in order to correctly determine the License plate number of the vehicle.

Although a lot of work has been done (as evident from the literature survey), yet there are open issues as discussed. Synergetic approaches from researchers need to converge on these issues so that the ALPR system may become error free and can be used as reliable systems.

\section{REFERENCES}

[1] Garima R. Yadav, H.K. Waghmare, "Automatic Indian License Plate Recognition system," IJARCSSE, Vol.4, Issue 11, November 2014.

[2] Pooya Sagharichi Ha, Majtaba Shakeri, "License Plate Automatic Recognition based on Edge Detection," unpublished.

[3] Amninder Kaur, Sonika Jindal, Richa Jindal, "License Plate Recognition using Support Vector Machine (SVM)," IJARCSSE, Vol. 2, Issue 7, July 2012.

[4] Kumar Parasuraman, Subin P.S, "SVM Based License Plate Recognition System," IEEE International Conference on Computational Intelligence and Computig Research, Tamilnadu College of Engineering Coimbatore, Dec 2010.
[5] Harpreet Kaur, Naresh Kumar Garg, "Number Plate Recognition Using Neural Network Classifier and KMEAN,” IJARCSSE, Vol. 4, Issue 8, August 2014.

[6] Jitender Kumar, Pawan Kumar Dahiya, "Automatic number plate recognition: a state-of-the-art", the World congress on Engineering 2014 (WCE 2014) London, U.K., july, 2014.

[7] Muhammad H Dashtban, Zahra Dashtban, Hassan Bevrani, "A Novel Approach For Vehicle License Plate Localization and Recognition," International Journal of Computer Applications, Vol. 26, No. 11, July 2012.

[8] Ronak P Patel, Narendra M Patel, Keyur Brahmbhatt, "Automatic License Plate Recognition," IJCSMC, Vol. 2, Issue 4, April 2013, pages. 285-294.

[9] M. M. Shidore, S. P. Narote, "Number Plate Recognition For Indian Vehicles," IJCSNS, Vol. 11, No. 2, Feb 2011.

[10] Sheetal Rani, Pawan Kumar Dahiya, "A Review of Recognition Technique Used Automatic License Plate Recognition System," International Journal of Computer Applications (0975-8887), Vol. 121, No.17, July 2015.

[11] B. Y. Amirgaliyev, C. A. Kenshimov, K. K. Kuatov, M. Z.Kairanbay, Z.Y. Baibatyr, A. K. Jantassov, "License Plate Verification Method For Automatic License Plate Recognition Systems," unpublished.

[12] Simon Tong, Daphne Koller, "Support Vector Machine Active Learning with Applications to Text Classification," Journal of Machine Learning Research (2001) 45-66

[13] Priti Rajvanshi, “Automatic Number Plate RecognitionApproach for Detecting the Vehicle Number Plate OnThe-Go," International Journal of Advanced Networking Applications, Special Conference Issue: National Conference on Cloud Computing \& Big Data, pages. 83-89.

[14] Imran Shafiq Ahmad, Boubakeur Boufama, Pejman Habashi, William Anderson, Tarik Elamsy, "Automatic License Plate Recognition: A Comparitive Study,” IEEE International Symposium on Signal Processing and Information Technology (ISSPIT), Abu Dhabi, UAE, Dec 2015, pages 635-640

[15] Anisha Goyal, Rekha Bhatia, "Automated Car Number Plate Detection System to Detect Far Number Plates," IOSR Journal of Computer Engineering, Vol. 18, Issue 4, Ver. III (Jul-Aug 2016), PP 34-40.

[16] Sahar S. Tabrizi, Nadire Cavus,"A hybrid KNN-SVM model for Iranian license plate recognition," 12th International Conference on Application of Fuzzy Systems and Soft Computing, ICAFS 2016, Vienna, Austria, 29-30 August 2016, pages 588-594.

[17] Sweta Kumari, Leeza Gupta, Prerna Gupta, "Automatic License Plate Recognition Using OpenCV and Neural Network," International Journal of Computer Science Trends and Technology (IJCST), Volume 5 Issue 3, May-Jun 2017, pages 114-118.

[18] Prof.R.M.Sahu, Namrata B.Gaikwad, Chandrakant B.Sandage, Vikram S.Shinde, "IoT Based Automatic Vehicle License Plate Recognition System," IJARIIE, Vol-3 Issue-2 2017, pages 3552-3558. 